\title{
Horadam polynomials and their applications to new family of bi-univalent functions with respect to symmetric conjugate points
}

Abbas Kareem Wanas ${ }^{1}$ (10 orcid.org/0000-0001-5838-7365 Sibel Yalçın² [0 orcid.org/0000-0002-0243-8263

${ }^{1}$ University of Al-Qadisiyah, Dept. of Mathematics, Al Diwaniyah, Qadisiyyah, Iraq.

abbas.kareem.w@qu.edu.iq

${ }^{2}$ Bursa Uludağ University, Dept. of Mathematics, Bursa, Turkey.

- syalcin@uludag.edu.tr

Received: January 2020 | Accepted: July 2020

\section{Abstract:}

In the current paper, by making use of the Horadam polynomials, we introduce and investigate a new family of holomorphic and biunivalent functions with respect to symmetric conjugate points defined in the open unit disk $D$. We derive upper bounds for the second and third coefficients and solve Fekete-Szegö problem of functions belongs to this family.

Keywords: Bi-univalent function; Horadam polynomials; Upper bounds; Symmetric conjugate; Fekete-Szegö problem; Subordination.

MSC (2020): 30C45, 30C50.

\section{Cite this article as (IEEE citation style):}

A. K. Wanas and S. Yalçın, "Horadam polynomials and their applications to new family of bi-univalent functions with res-

pect to symmetric conjugate points", Proyecciones (Antofagasta, On line), vol. 40, no. 1, pp. 106-116, 2021, doi: 10.22199/issn.0717-6279-2021-01-0007

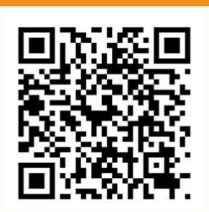

Article copyright: (C) 2021 Abbas Kareem Wanas and Sibel Yalçı. This is an open access article distributed under the terms of the Creative Commons License, which permits unrestricted use and distribution provided the original author and source are credited. 


\section{Introduction}

Denote by $\mathcal{A}$ the collection of holomorphic functions in the open unit disk $\mathbf{D}=\{z \in \mathbf{C}:|z|<1\}$ that have the form:

$$
f(z)=z+\sum_{n=2}^{\infty} a_{n} z^{n}
$$

Further, let $S$ indicate the sub-collection of $\mathcal{A}$ consisting of functions in D satisfying (1.1) which are univalent in $\mathbf{D}$.

Also, let $S_{s c}^{*}$ be the subclass of $S$ consisting of functions given by (1.1) satisfying

$$
\operatorname{Re}\left\{\frac{z f^{\prime}(z)}{f(z)-\overline{f(-\bar{z})}}\right\}>0, \quad z \in \mathbf{D} .
$$

These functions are called starlike with respect to symmetric conjugate points and were introduced by El-Ashwah and Thomas [6]. The class can be extended to other class in $\mathbf{D}$, namely convex functions with respect to symmetric conjugate points. Let $C_{s c}$ denote the class of convex functions with respect to symmetric conjugate points and satisfy the conditions

$$
\operatorname{Re}\left\{\frac{\left(z f^{\prime}(z)\right)^{\prime}}{(f(z)-\overline{f(-\bar{z})})^{\prime}}\right\}>0, \quad z \in \mathbf{D} .
$$

According to the Koebe One-Quarter Theorem [5] "every function $f \in S$ has an inverse $f^{-1}$ defined by $f^{-1}(f(z))=z,(z \in \mathbf{D})$ and $f\left(f^{-1}(w)\right)=w$, $\left(|w|<r_{0}(f), r_{0}(f) \geq \frac{1}{4}\right) "$, where

$$
g(w)=f^{-1}(w)=w-a_{2} w^{2}+\left(2 a_{2}^{2}-a_{3}\right) w^{3}-\left(5 a_{2}^{3}-5 a_{2} a_{3}+a_{4}\right) w^{4}+\cdots .
$$

A function $f \in \mathcal{A}$ is said to be bi-univalent in $\mathbf{D}$ if both $f$ and $f^{-1}$ are univalent in $\mathbf{D}$. Let $\Sigma$ stands for the class of bi-univalent functions in D given by (1.1). In fact, Srivastava et al. [16] has apparently revived the study of holomorphic and bi-univalent functions in recent years, it was followed by such works as those by Bulut [4], Altınkaya and Yalçın [2, 3], Adegani et al. [1] and others (see, for example [13, 14, 15, 17, 18, 19]). We notice that the class $\Sigma$ is not empty. For example, the functions $z$, $\frac{z}{1-z},-\log (1-z)$ and $\frac{1}{2} \log \frac{1+z}{1-z}$ are members of $\Sigma$. However, the Koebe function is not a member of $\Sigma$. Until now, the coefficient estimate problem 
for each of the following Taylor-Maclaurin coefficients $\left|a_{n}\right|,(n=3,4, \cdots)$ for functions $f \in \Sigma$ is still an open problem.

"With a view to recalling the principal of subordination between holomorphic functions, let the functions $f$ and $g$ be holomorphic in $\mathbf{D}$. We say that the function $f$ is said to be subordinate to $g$, if there exists a Schwarz function $w$ holomorphic in $\mathbf{D}$ with $w(0)=0$ and $|w(z)|<1(z \in \mathbf{D})$ such that $f(z)=g(w(z))$. This subordination is denoted by $f \prec g$ or $f(z) \prec g(z)$ $(z \in \mathbf{D})$. It is well known that (see [12]), if the function $g$ is univalent in $\mathbf{D}$, then $f \prec g$ if and only if $f(0)=g(0)$ and $f(\mathbf{D}) \subset g(\mathbf{D})$ ".

The Horadam polynomials $h_{n}(r)$ are defined by the following repetition relation (see [8]):

$$
h_{n}(r)=p r h_{n-1}(r)+q h_{n-2}(r) \quad(r \in \mathbf{R}, n \in \mathbf{N}=\{1,2,3, \cdots\}),
$$

with $h_{1}(r)=a \quad$ and $\quad h_{2}(r)=b r$, for some real constant $a, b, p$ and $q$. The characteristic equation of repetition relation (1.3) is $t^{2}-p r t-q=0$. This equation has two real roots $x=\frac{p r+\sqrt{p^{2} r^{2}+4 q}}{2}$ and $y=\frac{p r-\sqrt{p^{2} r^{2}+4 q}}{2}$.

Remark 1.1. By selecting the particular values of $a, b, p$ and $q$, the Horadam polynomial $h_{n}(r)$ reduces to several polynomials. Some of them are illustrated below:

1. Taking $a=b=p=q=1$, we obtain the Fibonacci polynomials $F_{n}(r)$.

2. Taking $a=2$ and $b=p=q=1$, we attain the Lucas polynomials $L_{n}(r)$.

3. Taking $a=q=1$ and $b=p=2$, we have the Pell polynomials $P_{n}(r)$.

4. Taking $a=b=p=2$ and $q=1$, we get the Pell-Lucas polynomials $Q_{n}(r)$.

5. Taking $a=b=1, p=2$ and $q=-1$, we obtain the Chebyshev polynomials $T_{n}(r)$ of the first kind.

6. Taking $a=1, b=p=2$ and $q=-1$, we have the Chebyshev polynomials $U_{n}(r)$ of the second kind. 
These polynomials, the families of orthogonal polynomials and other special polynomials as well as their generalizations are potentially important in a variety of disciplines in many of sciences, specially in the mathematics, statistics and physics. For more information associated with these polynomials see $[7,8,10,11]$.

The generating function of the Horadam polynomials $h_{n}(r)$ (see [9]) is given by

$$
\Pi(r, z)=\sum_{n=1}^{\infty} h_{n}(r) z^{n-1}=\frac{a+(b-a p) r z}{1-p r z-q z^{2}} .
$$

\section{Main Results}

We begin this section by defining the family $\mathcal{G}_{\Sigma}(\lambda, \eta, r)$ as follows:

Definition 2.1. For $0 \leq \eta \leq \lambda \leq 1$ and $r \in \mathbf{R}$, a function $f \in \Sigma$ with $a_{n} \in \mathbf{R}$ is said to be in the class $\mathcal{G}_{\Sigma}(\lambda, \eta, r)$ if it fulfills the subordinations:

$$
\begin{gathered}
\frac{2\left[\lambda \eta z^{3} f^{\prime \prime \prime}(z)+(\lambda+\eta(2 \lambda-1)) z^{2} f^{\prime \prime}(z)+z f^{\prime}(z)\right]}{\lambda \eta z^{2}(f(z)-\overline{f(-\bar{z})})^{\prime \prime}+(\lambda-\eta) z(f(z)-\overline{f(-\bar{z})})^{\prime}+(1-\lambda+\eta)(f(z)-\overline{f(-\bar{z})})} \\
\prec \Pi(r, z)+1-a
\end{gathered}
$$

and

$$
\begin{gathered}
\frac{2\left[\lambda \eta w^{3} g^{\prime \prime \prime}(w)+(\lambda+\eta(2 \lambda-1)) w^{2} g^{\prime \prime}(w)+w g^{\prime}(w)\right]}{\lambda \eta w^{2}(g(w)-\overline{g(-\bar{w})})^{\prime \prime}+(\lambda-\eta) w(g(w)-\overline{g(-\bar{w})})^{\prime}+(1-\lambda+\eta)(g(w)-\overline{g(-\bar{w})})} \\
\prec \Pi(r, w)+1-a,
\end{gathered}
$$

where $a$ is real constant and the function $g=f^{-1}$ is given by (1.2).

Theorem 2.1. For $0 \leq \eta \leq \lambda \leq 1$ and $r \in \mathbf{R}$, let $f \in \mathcal{A}$ with $a_{n} \in \mathbf{R}$ be in the class $\mathcal{G}_{\Sigma}(\lambda, \eta, r)$. Then

$$
\left|a_{2}\right| \leq \frac{|b r| \sqrt{|b r|}}{\sqrt{2\left|\left[(6 \lambda \eta+2(\lambda-\eta)+1) b-2 p(2 \lambda \eta+\lambda-\eta+1)^{2}\right] b r^{2}-2 q a(2 \lambda \eta+\lambda-\eta+1)^{2}\right|}}
$$

and

$$
\left|a_{3}\right| \leq \frac{|b r|}{2(6 \lambda \eta+2(\lambda-\eta)+1)}+\frac{b^{2} r^{2}}{4(2 \lambda \eta+\lambda-\eta+1)^{2}}
$$


Proof. Let $f \in \mathcal{G}_{\Sigma}(\lambda, \eta, r)$. Then there are two holomorphic functions $u, v: \mathbf{D} \longrightarrow \mathbf{D}$ given by

$$
u(z)=u_{1} z+u_{2} z^{2}+u_{3} z^{3}+\cdots \quad(z \in \mathbf{D})
$$

and

$$
v(w)=v_{1} w+v_{2} w^{2}+v_{3} w^{3}+\cdots \quad(w \in \mathbf{D}),
$$

with $u(0)=v(0)=0,|u(z)|<1,|v(w)|<1, z, w \in \mathbf{D}$ such that

$$
\begin{gathered}
\frac{2\left[\lambda \eta z^{3} f^{\prime \prime \prime}(z)+(\lambda+\eta(2 \lambda-1)) z^{2} f^{\prime \prime}(z)+z f^{\prime}(z)\right]}{\lambda \eta z^{2}(f(z)-\overline{f(-\bar{z})})^{\prime \prime}+(\lambda-\eta) z(f(z)-\overline{f(-\bar{z})})^{\prime}+(1-\lambda+\eta)(f(z)-\overline{f(-\bar{z})})} \\
=\Pi(r, u(z))+1-a
\end{gathered}
$$

and

$$
\begin{gathered}
\frac{2\left[\lambda \eta w^{3} g^{\prime \prime \prime}(w)+(\lambda+\eta(2 \lambda-1)) w^{2} g^{\prime \prime}(w)+w g^{\prime}(w)\right]}{\lambda \eta w^{2}(g(w)-\overline{g(-\bar{w})})^{\prime \prime}+(\lambda-\eta) w(g(w)-\overline{g(-\bar{w})})^{\prime}+(1-\lambda+\eta)(g(w)-\overline{g(-\bar{w})})} \\
=\Pi(r, v(w))+1-a .
\end{gathered}
$$

Or, equivalently

$$
\begin{aligned}
& \frac{2\left[\lambda \eta z^{3} f^{\prime \prime \prime}(z)+(\lambda+\eta(2 \lambda-1)) z^{2} f^{\prime \prime}(z)+z f^{\prime}(z)\right]}{\lambda \eta z^{2}(f(z)-\overline{f(-\bar{z})})^{\prime \prime}+(\lambda-\eta) z(f(z)-\overline{f(-\bar{z})})^{\prime}+(1-\lambda+\eta)(f(z)-\overline{f(-\bar{z})})} \\
& (2.3) \quad=1+h_{1}(r)+h_{2}(r) u(z)+h_{3}(r) u^{2}(z)+\cdots
\end{aligned}
$$

and

$$
\begin{aligned}
& \frac{2\left[\lambda \eta w^{3} g^{\prime \prime \prime}(w)+(\lambda+\eta(2 \lambda-1)) w^{2} g^{\prime \prime}(w)+w g^{\prime}(w)\right]}{\lambda \eta w^{2}(g(w)-\overline{g(-\bar{w})})^{\prime \prime}+(\lambda-\eta) w(g(w)-\overline{g(-\bar{w})})^{\prime}+(1-\lambda+\eta)(g(w)-\overline{g(-\bar{w})})} \\
& (2.4) \quad=1+h_{1}(r)+h_{2}(r) v(w)+h_{3}(r) v^{2}(w)+\cdots .
\end{aligned}
$$

Combining (2.1), (2.2), (2.3) and (2.4) yields

$$
\frac{2\left[\lambda \eta z^{3} f^{\prime \prime \prime}(z)+(\lambda+\eta(2 \lambda-1)) z^{2} f^{\prime \prime}(z)+z f^{\prime}(z)\right]}{\lambda \eta z^{2}(f(z)-\overline{f(-\bar{z})})^{\prime \prime}+(\lambda-\eta) z(f(z)-\overline{f(-\bar{z})})^{\prime}+(1-\lambda+\eta)(f(z)-\overline{f(-\bar{z})})}
$$




$$
=1+h_{2}(r) u_{1} z+\left[h_{2}(r) u_{2}+h_{3}(r) u_{1}^{2}\right] z^{2}+\cdots
$$

and

$\frac{2\left[\lambda \eta w^{3} g^{\prime \prime \prime}(w)+(\lambda+\eta(2 \lambda-1)) w^{2} g^{\prime \prime}(w)+w g^{\prime}(w)\right]}{\lambda \eta w^{2}(g(w)-\overline{g(-\bar{w})})^{\prime \prime}+(\lambda-\eta) w(g(w)-\overline{g(-\bar{w})})^{\prime}+(1-\lambda+\eta)(g(w)-\overline{g(-\bar{w})})}$

$$
=1+h_{2}(r) v_{1} w+\left[h_{2}(r) v_{2}+h_{3}(r) v_{1}^{2}\right] w^{2}+\cdots .
$$

It is quite well-known that if $|u(z)|<1$ and $|v(w)|<1, z, w \in \mathbf{D}$, then

$$
\left|u_{i}\right| \leq 1 \quad \text { and } \quad\left|v_{i}\right| \leq 1 \text { for all } i \in \mathbf{N} .
$$

Comparing the corresponding coefficients in (2.5) and (2.6), after simplifying, we have

$$
\begin{gathered}
2(2 \lambda \eta+\lambda-\eta+1) a_{2}=h_{2}(r) u_{1}, \\
2(6 \lambda \eta+2(\lambda-\eta)+1) a_{3}=h_{2}(r) u_{2}+h_{3}(r) u_{1}^{2}, \\
-2(2 \lambda \eta+\lambda-\eta+1) a_{2}=h_{2}(r) v_{1}
\end{gathered}
$$

and

$$
2(6 \lambda \eta+2(\lambda-\eta)+1)\left(2 a_{2}^{2}-a_{3}\right)=h_{2}(r) v_{2}+h_{3}(r) v_{1}^{2} .
$$

In view of (2.8) and (2.10), we conclude that

$$
u_{1}=-v_{1}
$$

and

$$
8(2 \lambda \eta+\lambda-\eta+1)^{2} a_{2}^{2}=h_{2}^{2}(r)\left(u_{1}^{2}+v_{1}^{2}\right) .
$$

If we add (2.9) to (2.11), we find that

$$
4(6 \lambda \eta+2(\lambda-\eta)+1) a_{2}^{2}=h_{2}(r)\left(u_{2}+v_{2}\right)+h_{3}(r)\left(u_{1}^{2}+v_{1}^{2}\right) .
$$

Substituting the value of $u_{1}^{2}+v_{1}^{2}$ from (2.13) into (2.14), it follows that 


$$
a_{2}^{2}=\frac{h_{2}^{3}(r)\left(u_{2}+v_{2}\right)}{4\left[h_{2}^{2}(r)(6 \lambda \eta+2(\lambda-\eta)+1)-2 h_{3}(r)(2 \lambda \eta+\lambda-\eta+1)^{2}\right]}
$$

Further computations using (1.3), (2.7) and (2.15), we deduce that

$$
\left|a_{2}\right| \leq \frac{|b r| \sqrt{|b r|}}{\sqrt{2\left|\left[(6 \lambda \eta+2(\lambda-\eta)+1) b-2 p(2 \lambda \eta+\lambda-\eta+1)^{2}\right] b r^{2}-2 q a(2 \lambda \eta+\lambda-\eta+1)^{2}\right|}} .
$$

To determinate the bound on $\left|a_{3}\right|$, by subtracting (2.11) from (2.9), we can easily see that

$$
4(6 \lambda \eta+2(\lambda-\eta)+1)\left(a_{3}-a_{2}^{2}\right)=h_{2}(r)\left(u_{2}-v_{2}\right)+h_{3}(r)\left(u_{1}^{2}-v_{1}^{2}\right)
$$

Also, by using (2.12) and (2.13) together with (2.16), we conclude that

$$
a_{3}=\frac{h_{2}(r)\left(u_{2}-v_{2}\right)}{4(6 \lambda \eta+2(\lambda-\eta)+1)}+\frac{h_{2}^{2}(r)\left(u_{1}^{2}+v_{1}^{2}\right)}{8(2 \lambda \eta+\lambda-\eta+1)^{2}} .
$$

Thus applying (1.3), we obtain

$$
\left|a_{3}\right| \leq \frac{|b r|}{2(6 \lambda \eta+2(\lambda-\eta)+1)}+\frac{b^{2} r^{2}}{4(2 \lambda \eta+\lambda-\eta+1)^{2}} .
$$

This completes the proof of Theorem 2.1

In the next theorem, we discuss the "Fekete-Szegö problem" for the family $\mathcal{G}_{\Sigma}(\lambda, \eta, r)$.

Theorem 2.2. For $0 \leq \eta \leq \lambda \leq 1$ and $r, \mu \in \mathbf{R}$, let $f \in \mathcal{A}$ with $a_{n} \in \mathbf{R}$ be in the family $\mathcal{G}_{\Sigma}(\lambda, \eta, r)$. Then

$$
\left|a_{3}-\mu a_{2}^{2}\right| \leq\left\{\begin{array}{c}
\frac{|b r|}{2(6 \lambda \eta+2(\lambda-\eta)+1)} \\
\text { for }|\mu-1| \leq \frac{\left|\left[(6 \lambda \eta+2(\lambda-\eta)+1) b-2 p(2 \lambda \eta+\lambda-\eta+1)^{2}\right] b r^{2}-2 q a(2 \lambda \eta+\lambda-\eta+1)^{2}\right|}{b^{2} r^{2}(6 \lambda \eta+2(\lambda-\eta)+1)} \\
\frac{|b r|^{3}|\mu-1|}{2\left|\left[(6 \lambda \eta+2(\lambda-\eta)+1) b-2 p(2 \lambda \eta+\lambda-\eta+1)^{2}\right] b r^{2}-2 q a(2 \lambda \eta+\lambda-\eta+1)^{2}\right|} \\
\text { for }|\mu-1| \geq \frac{\left|\left[(6 \lambda \eta+2(\lambda-\eta)+1) b-2 p(2 \lambda \eta+\lambda-\eta+1)^{2}\right] b r^{2}-2 q a(2 \lambda \eta+\lambda-\eta+1)^{2}\right|}{b^{2} r^{2}(6 \lambda \eta+2(\lambda-\eta)+1)}
\end{array}\right.
$$


Proof. In the light of (2.15) and (2.16), we find that

$$
\begin{aligned}
a_{3}-\mu a_{2}^{2} & =\frac{h_{2}(r)\left(u_{2}-v_{2}\right)}{4(6 \lambda \eta+2(\lambda-\eta)+1)}+(1-\mu) a_{2}^{2} \\
& =\frac{h_{2}(r)\left(u_{2}-v_{2}\right)}{4(6 \lambda \eta+2(\lambda-\eta)+1)}+\frac{h_{2}^{3}(r)\left(u_{2}+v_{2}\right)(1-\mu)}{4\left[h_{2}^{2}(r)(6 \lambda \eta+2(\lambda-\eta)+1)-2 h_{3}(r)(2 \lambda \eta+\lambda-\eta+1)^{2}\right]} \\
& =\frac{h_{2}(r)}{4}\left[\left(\psi(\mu, r)+\frac{1}{(6 \lambda \eta+2(\lambda-\eta)+1)}\right) u_{2}+\left(\psi(\mu, r)-\frac{1}{(6 \lambda \eta+2(\lambda-\eta)+1)}\right) v_{2}\right],
\end{aligned}
$$

where

$$
\psi(\mu, r)=\frac{h_{2}^{2}(r)(1-\mu)}{h_{2}^{2}(r)(6 \lambda \eta+2(\lambda-\eta)+1)-2 h_{3}(r)(2 \lambda \eta+\lambda-\eta+1)^{2}} .
$$

According to (1.3), we deduce that

$$
\left|a_{3}-\mu a_{2}^{2}\right| \leq\left\{\begin{array}{lr}
\frac{|b r|}{2(6 \lambda \eta+2(\lambda-\eta)+1)}, & 0 \leq|\psi(\mu, r)| \leq \frac{1}{6 \lambda \eta+2(\lambda-\eta)+1} \\
\frac{1}{2}|b r||\psi(\mu, r)|, & |\psi(\mu, r)| \geq \frac{1}{6 \lambda \eta+2(\lambda-\eta)+1}
\end{array}\right.
$$

After some computations, we obtain

$$
\left|a_{3}-\mu a_{2}^{2}\right| \leq\left\{\begin{array}{c}
\frac{|b r|}{2(6 \lambda \eta+2(\lambda-\eta)+1)} \\
\text { for }|\mu-1| \leq \frac{\left|\left[(6 \lambda \eta+2(\lambda-\eta)+1) b-2 p(2 \lambda \eta+\lambda-\eta+1)^{2}\right] b r^{2}-2 q a(2 \lambda \eta+\lambda-\eta+1)^{2}\right|}{b^{2} r^{2}(6 \lambda \eta+2(\lambda-\eta)+1)} \\
\frac{|b r|^{3}|\mu-1|}{2\left|\left[(6 \lambda \eta+2(\lambda-\eta)+1) b-2 p(2 \lambda \eta+\lambda-\eta+1)^{2}\right] b r^{2}-2 q a(2 \lambda \eta+\lambda-\eta+1)^{2}\right|} \\
\text { for }|\mu-1| \geq \frac{\left|\left[(6 \lambda \eta+2(\lambda-\eta)+1) b-2 p(2 \lambda \eta+\lambda-\eta+1)^{2}\right] b r^{2}-2 q a(2 \lambda \eta+\lambda-\eta+1)^{2}\right|}{b^{2} r^{2}(6 \lambda \eta+2(\lambda-\eta)+1)} .
\end{array}\right.
$$

Putting $\mu=1$ in Theorem 2.2, we obtain the following result:

Corollary 2.1. For $0 \leq \eta \leq \lambda \leq 1$ and $r \in \mathbf{R}$, let $f \in \mathcal{A}$ be in the family $\mathcal{G}_{\Sigma}(\lambda, \eta, r)$. Then

$$
\left|a_{3}-a_{2}^{2}\right| \leq \frac{|b r|}{2(6 \lambda \eta+2(\lambda-\eta)+1)}
$$




\section{References}

[1] E. A. Adegani, S. Bulut, and A. A. Zireh, "Coefficient estimates for a subclass of analytic bi-univalent functions", Bulletin Korean Mathematical Society, vol. 55, no. 2, pp. 405-413, 2018, doi: 10.4134/ BKMS.b170051

[2] S.. Altınkaya and S. Yalçın, "On the Chebyshev coefficients for a general subclass of univalent functions", Turkish journal of mathematics, vol. 42, no. 6, pp. 2885-2890, 2018, doi: 10.3906/ mat-1510-53

[3] Ş. Altınkaya and S. Yalçın, "On the Chebyshev polynomial coefficient problem of some subclasses of bi-univalent functions", Gulf journal of mathematics, vol. 5, no. 3, pp. 34-40, 2017. [On line]. Available: https:/ / bit.ly/ 2JOLqwa

[4] S. Bulut, "Coefficient estimates for general subclasses of m-fold symmetric analytic bi-univalent functions", Turkish journal of mathematics, vol. 40, no. 6, pp. 1386-1397, 2016, doi: 10.3906/ mat-1511-41

[5] P. L. Duren, Univalent functions. New York, NY: Springer, 1983.

[6] R. M. El-Ashwah and D. K. Thomas, "Some subclasses of close-to-convex functions", Journal of the Ramanujan Mathematical Society, vol. 2, no. 1, pp. 86-100, 1987. [On line]. Available: https:/ / bit.ly/ 3bm41eF

[7] A. F. Horadam, "Jacobsthal representation polynomials", The Fibonacci quarterly, vol. 35, no.2, pp. 137-148, 1997. [On line]. Available: https:/ / bit.ly/2L1Slmy

[8] A. F. Horadam and J. M. Mahon, "Pell and Pell-Lucas polynomials", The Fibonacci quarterly, vol. 23, no. 1, pp. 7-20, 1985. [On line]. Available: https:/ / bit.ly/2XeKfJC

[9] T. Horzum and E. G. Kocer, "On some properties of Horadam polynomials", International mathematical forum, vol. 4, no. 25, pp. 12431252, 2009. [On line]. Available: https:// bit.ly/3npQHYS

[10] T. Koshy, Fibonacci and Lucas numbers with applications. New York, NY: A Wiley-Interscience, 2001, doi: 10.1002/9781118033067 
[11] A. Lupas, "A Guide of Fibonacci and Lucas polynomials", Octagon mathematics magazine, vol. 7, no. 1, pp. 2-12, 1999.

[12] S. S. Miller and P. Mocanu, Differential subordinations: theory and applications. New York, NY: Marcel Dekker, 2000.

[13] H. M. Srivastava, S. Altınkaya, and S. Yalçın, "Certain subclasses of bi-univalent functions associated with the Horadam polynomials", Iranian journal of science and technology, transactions A: Science, vol. 43, pp. 1873-1879, 2019, doi: 10.1007/ s40995-018-0647-0

[14] H. M. Srivastava, S. S. Eker, S. G. Hamidi, and J. M. Jahangiri, "Faber polynomial coefficient estimates for bi-univalent functions defined by the Tremblay fractional derivative operator", Bulletin Iranian Mathematical Society, vol. 44, no. 1, pp. 149-157, 2018, doi: 10.1007/ s41980- 018-0011-3

[15] H. M. Srivastava, S. Gaboury, and F. Ghanim, "Coefficient estimates for some general subclasses of analytic and bi-univalent functions", Afrika matematika, vol. 28, pp. 693-706, 2017, doi: 10.1007/ s13370-016-0478-0

[16] H. M. Srivastava, A. K. Mishra, and P. Gochhayat, "Certain subclasses of analytic and Bi-Univalent functions", Applied mathematics letters, vol. 23, pp. 1188-1192, 2010, doi: 10.1016/j.aml.2010.05.009

[17] H. M. Srivastava and A. K. Wanas, "Initial Maclaurin coefficient bounds for new subclasses of analytic and $\mathrm{m}$-fold symmetric bi-univalent functions defined by a linear combination", Kyungpook mathematical journal, vol. 59, no. 3, pp. 493-503, 2019, doi: 10.5666/ KMJ.2019.59.3.493

[18] A. K. Wanas and A. L. Alina, "Applications of Horadam polynomials on Bazilevič bi-univalent function satisfying subordinate conditions", Journal of physics: conference series, vol. 1294, no. 3, Art ID. 032003, 2019 doi: 10.1088/ 1742-6596/ 1294/3/ 032003

[19] A. K. Wanas and S. Yalçın, "Initial coefficient estimates for a new subclasses of analytic and m-Fold symmetric bi-univalent functions", Malaya journal of matematik, vol. 7, no. 3, pp. 472-476, 2019, doi: 10.26637/ MJM0703/ 0018 